\title{
Level Diagnosis of Cervical Compressive Myelopathy: Signs, Symptoms, and Lesions Levels
}

\author{
Naoki Kasahata
}

\begin{abstract}
Background: To elucidate signs and symptoms corresponding to each vertebral level for level-specific diagnoses.

Methods: We studied 106 patients with cervical compressive myelopathy. Patients who showed a single compressive site on magnetic resonance imaging (MRI) were selected, and signs, symptoms, and the levels of the MRI lesions were studied.
\end{abstract}

Results: Five of 12 patients (41.7\%) with C4-5 intervertebral level lesions showed decreased or absent biceps and brachioradialis reflexes, while 4 of these patients (33.3\%) showed generalized hyperreflexia. In comparison, 5 of 24 patients (20.8\%) with C5-6 intervertebral level lesions showed decreased or absent triceps reflexes; however, 9 of these patients $(37.5 \%)$ showed decreased or absent biceps and brachioradialis reflexes.

Conclusions: C4-5 intervertebral level lesions not only showed C6 segment signs but also showed more rostral segment signs. Similarly, C5-6 intervertebral level lesions not only showed C7 segment signs but also showed C6 or more rostral segment signs.

Keywords: Cervical compressive myelopathy; Level diagnosis; Magnetic resonance imaging

\section{Introduction}

Myelopathy has been a disease with multiple presentations, with variable severity at the time of diagnosis, making it dif-

Manuscript accepted for publication October 15, 2013

Department of Medicine, Division of Neurology, Tokyo Metropolitan Ohtsuka Hospital, 2-8-1 Minamiohtsuka, Toshima-ku, Tokyo 170-8476, Japan.Email: n_kasa-o@ohtsuka-hospital.toshima.tokyo.jp

doi: http://dx.doi.org/10.4021/jnr236w ficult to accurately localize the lesion before radiographic diagnosis. However, neurological level diagnosis of spinal cord is important for accurate lesion-specific level diagnosis, patients' treatment, avoiding diagnostic error, differential diagnosis, and especially for accurate level diagnosis of other nonsurgical myelopathies. Moreover, level diagnosis should be considered from multiple viewpoints. Therefore, we intend to make level diagnosis of myelopathy more accurate.

Previously, lesion-specific level diagnoses by determining a sensory disturbance area or location of numbness in the hands had the highest accuracy $[1,2]$. Previous studies reported that C3-4 intervertebral level lesions showed increased or decreased biceps reflexes, deltoid weakness, and sensory disturbance of arms or forearms [1, 3, 4], while C4-5 intervertebral level lesions showed decreased biceps reflexes, biceps weakness, and sensory disturbance of hands $[1,3,4]$. Moreover, these studies reported that C5-6 intervertebral level lesions showed decreased triceps reflexes, triceps weakness, and sensory disturbance of ulnar side of the fingers $[1,3,4]$, C6-7 intervertebral level lesions showed similar results in approximately half of the cases but remaining half cases were normal $[1,3,4]$. Another study reported that C5-6 intervertebral level lesions manifested as dysesthesia of the thumb and index finger, with muscle stretch reflexes in the biceps and brachioradialis were diminished [5]. In contrast to C5-6 intervertebral level lesions, this study reported that C6-7 intervertebral level lesions manifested as dysesthesia of the middle finger with deep tendon reflexes in the triceps were diminished [5]. Patients with C3-4 intervertebral level lesions did not complain of dysesthesia, but muscle stretch reflexes were exaggerated in the upper extremities [5]. Moreover, bilateral finger jerks have been observed in patients with predominantly $\mathrm{C} 4-5$ or $\mathrm{C} 5-6$ intervertebral level lesions [6].

Cervical myelopathies with false localizing sensory levels have been reported [7, 8]. Numb and clumsy hands because of midline cervical disc protrusion at the $\mathrm{C} 3-4$ intervertebral level have also been reported [9]. Cervical angina, defined as chest pain, resembles a true cardiac angina but originates from a cervical discopathy and most commonly results from the compression of the $\mathrm{C} 7$ nerve root [10]. A wide-based spastic gait is the classical presentation of a 
Table 1. Clinical Characteristics of C4-5 Intervertebral Level Myelopathy

\begin{tabular}{|c|c|c|c|c|c|c|c|c|}
\hline Patient & Age/Sex & Biceps & Brachi & Triceps & PTR & Level & Tingling & Others \\
\hline 1 & $76 / \mathrm{F}$ & +++ & +++ & +++ & $++/+$ & - & Right L/E & Wartenberg + \\
\hline 2 & $66 / \mathrm{M}$ & +++ & +++ & +++ & +++ & - & - & Marche a petit pas \\
\hline 3 & $80 / \mathrm{M}$ & + & + & ++ & $-/-$ & $\mathrm{C} 5$ & - & Romberg + \\
\hline 4 & $74 / \mathrm{F}$ & + & + & +++ & +++ & C5-6 & - & Gait disturbance \\
\hline 5 & 64/M & ++ & ++ & ++ & +++ & - & - & Romberg \pm \\
\hline 6 & $55 / \mathrm{F}$ & +++ & ++ & ++ & +++ & - & Left hand & - \\
\hline 7 & $55 / \mathrm{M}$ & ++++ & ++++ & +++ & ++++ & C6 & - & Sacral sparing, Romberg \\
\hline 8 & $33 / \mathrm{M}$ & \pm & $+++/ \pm$ & $+/++$ & +++ & - & Rt 4 fing. & Finger jerks, Warten. + \\
\hline 9 & $41 / F$ & +++ & +++ & +++ & +++ & C6 & Left U/E & Finger jerk, hype. Lt U/E \\
\hline 10 & $46 / \mathrm{M}$ & ++ & ++ & Inv. & ++ & Th3 & Bil. thigh & Inverted reflex \\
\hline 11 & $62 / \mathrm{M}$ & \pm & + & ++ & ++ & - & Left hand & Finger jerks \\
\hline 12 & $76 / \mathrm{M}$ & - & - & ++ & - & - & Feet & Atrop. Lt 1DIO, Lt weak. \\
\hline
\end{tabular}

Brachi = brachioradialis; PTR = patellar tendon reflex; L/E = lower extremities; U/E = upper extremities; Bil = bilateral; Lt = left; Rt = right; fing. = fingers; Warten. = Wartenberg; hype. = hypesthesia; Inv. = inverted; Atrop. = atrophy; $\mathrm{DIO}=$ dorsal interosseous; weak. = weakness.

patient with cervical spondylotic myelopathy [11]. Moreover, positive Hoffmann's reflexes in asymptomatic patients strongly suggest underlining cervical pathology [12].

We studied the symptoms, signs, or magnetic resonance imaging (MRI) findings of 106 patients with cervical compressive myelopathy. Here, we report signs and symptoms of patients with single compressive sites, and discuss the level-specific diagnosis of cervical compressive myelopathy. We also describe patients with minimal change myelopathy, defined as a syndrome with signs and symptoms consistent with cervical compressive myelopathy without any apparent MRI lesions.

\section{Materials and Methods}

We studied symptoms, signs, and MRI findings of 106 patients with cervical compressive myelopathy as consecutive series from December 2008 to August 2011 in Tokyo Metropolitan Ohtsuka Hospital. The inclusion criteria were symptoms or signs consistent with cervical compressive myelopathy and diagnosed as cervical compressive myelopathy on the MRI findings. Approval of institutional human research review committee (Ethics Committee of Tokyo Metropolitan
Ohtsuka Hospital, Tokyo, Japan) was obtained. Intracranial diseases or neuropathies, such as compression neuropathy, were the differential diagnoses. Patients with intracranial diseases or neuropathies such as carpal tunnel syndrome, identified by brain MRI or nerve conduction study (NCS), were excluded from this study. Patients with factors that may cause mild derangement of peripheral nerve function (for example, diabetes mellitus and renal impairment) were also excluded.

Clinical characteristics, especially important for levelspecific diagnoses, such as muscle stretch reflexes findings of the upper extremities, sensory levels, localizations of tingling sensations, and other symptoms and signs were examined. The sensory level reported here refers to the level defined by pinprick sensation. Wartenberg reflex is one of finger flexor reflexes. Wartenberg reflex is the most sensitive among finger flexor reflexes such as Tromner or Hoffmann reflex. It may be false positive, and some normal persons may present with Wartenberg reflexes. When we examine triceps reflex of a patient with $\mathrm{C} 7$ segmental compression, a triceps reflex results in no contraction of triceps but contraction of biceps. This phenomenon is called inverted triceps reflex, which has been $\mathrm{C} 7$ segmental sign. When we examine brachioradialis reflex of a patient with $\mathrm{C} 6$ segmental compression, a bra- 

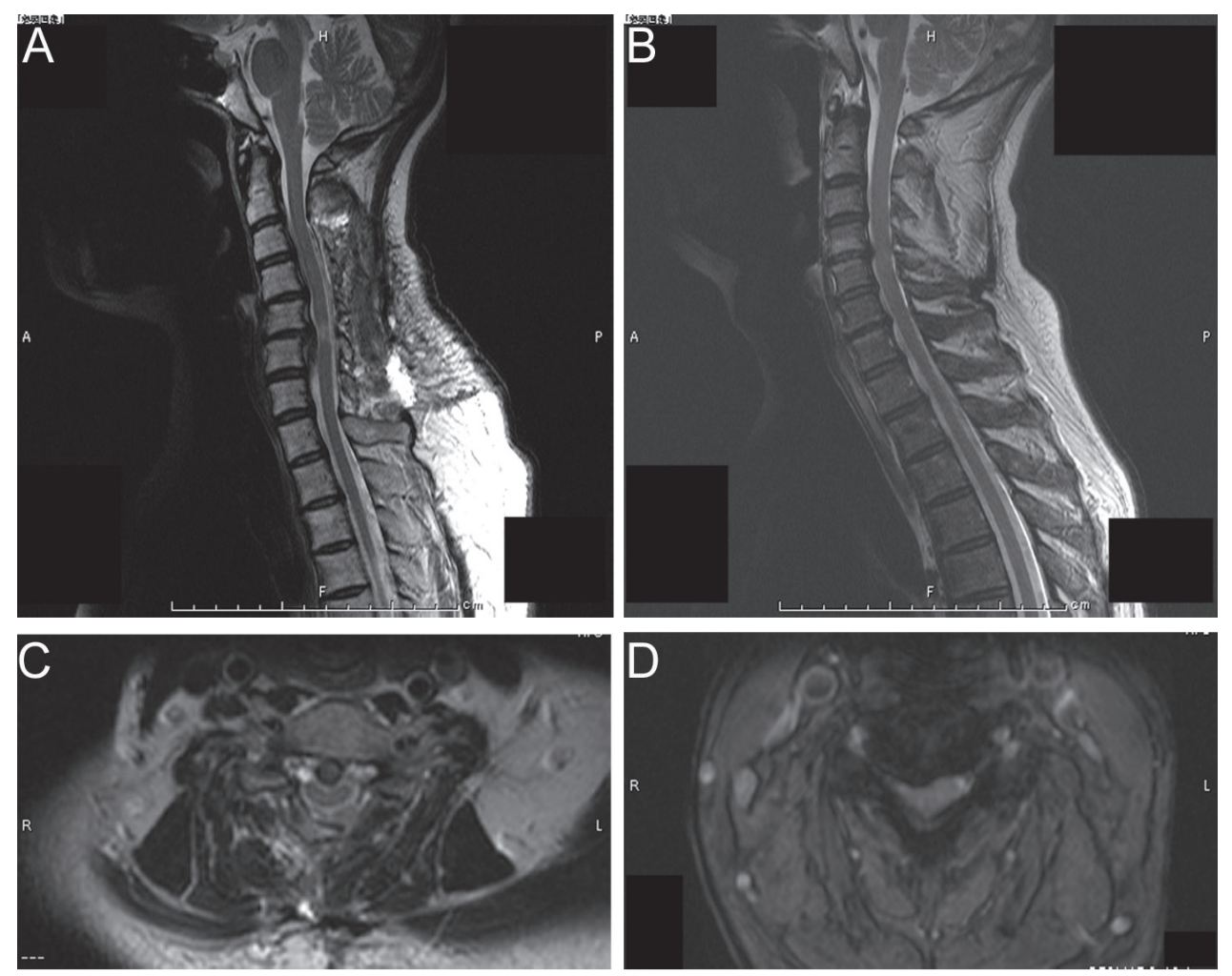

Figure 1. Sagittal and axial magnetic resonance imaging (MRI) findings of typical C5-6 and C4-5 intervertebral level myelopathies. (A) T2-weighted MRI of C5-6 intervertebral level myelopathy. Spinal cord corresponding to C5-6 intervertebral level shows a high intensity area (TR, 2400; TE, 120). (B) T2-weighted MRI of C4-5 intervertebral level myelopathy. Spinal cord corresponding to C4-5 intervertebral level is compressed (TR, 2400; TE, 120). (C) T2-weighted axial MRI corresponding to A (C5-6 intervertebral level). Spinal cord shows boomerang shaped deformity (TR, 2400; TE, 120). (D) T2-weighted axial MRI corresponding to B (C4-5 intervertebral level). Spinal cord shows right side dominant deformity (TR, 2400; TE, 120).

chioradialis reflex results in no contraction of brachioradialis but presented with finger jerks. This phenomenon is called inverted radial reflex, which has been C6 segment sign. Thirty-nine patients who showed a single compressive site on the MRI were selected, and signs, symptoms, and vertebral levels of the MRI lesions were compared. We excluded other 62 patients who showed two or more compressive sites on the MRI. Some patients clinically presented with cervical compressive myelopathy even though MRI showed no apparent lesions. These patients' clinical characteristics and MRI findings are summarized below.

\section{Results}

A summary of demographic information of all patients included as follows: mean age 66.3 (15-92); 66 men and 44 women. A summary of demographic information of 62 patients excluded from the analysis included as follows: mean age 71.7 (53-92); 44 men and 22 women; 2 compressive sites 31,3 compressive sites 14 , mild single compressive site 5 , ossification of posterior longitudinal ligament 1 (2 compres- sive sites), post-operative state of atlantoaxial dislocation 1 (3 compressive sites), and unavailable detailed information 12.

\section{C3-4 intervertebral level myelopathy}

Only 1 patient had a C3-4 intervertebral level lesion. This patient had generalized hyperreflexia, and her left hand dropped objects.

\section{C4-5 intervertebral level myelopathy}

Twelve patients had C4-5 intervertebral level lesions (4 women and 8 men). Five of these patients (41.7\%) showed decreased or absent biceps and brachioradialis reflexes, while $4(33.3 \%)$ showed generalized hyperreflexia. Three patients $(25 \%)$ showed finger jerks, which were elicited by the biceps and brachioradialis reflexes. Two of the 3 patients with finger jerks showed decreased biceps and brachioradialis reflexes (inverted radial reflexes), whereas the remaining 1 patient showed increased biceps and brachioradialis reflexes. Five patients $(41.7 \%)$ had sensory levels from C5 to Th3: C5 for 
Table 2. Clinical Characteristics of C5-6 Intervertebral Level Myelopathy

\begin{tabular}{|c|c|c|c|c|c|c|c|c|}
\hline Patient & Age/Sex & Biceps & Brachi & Triceps & PTR & Level & Tingling & Others \\
\hline 1 & $78 / \mathrm{F}$ & +++ & +++ & ++ & ++ & - & All E. & Hand atrophy \\
\hline 2 & $50 / \mathrm{F}$ & - & \pm & ++ & - & - & $\begin{array}{l}\text { All (right } \\
3-5)\end{array}$ & Romberg + \\
\hline 3 & $42 / \mathrm{M}$ & $++/-$ & $+/+++$ & ++ & ++ & - & $\mathrm{L} / \mathrm{E}$ & Wartenberg -/+ \\
\hline 4 & $66 / \mathrm{F}$ & +++ & +++ & +++ & +++ & $\mathrm{C} 7$ & Right U/E & - \\
\hline 5 & $69 / \mathrm{F}$ & +++ & +++ & Inv. & +++ & - & - & Finger jerks, Warten. + \\
\hline 6 & $69 / F$ & +++ & +++ & $++/ \pm$ & +++ & $\mathrm{C} 7$ & - & Finger weakness \\
\hline 7 & $88 / \mathrm{M}$ & - & - & + & ++ & - & - & Gait disturbance \\
\hline 8 & $54 / \mathrm{F}$ & - & - & $+/++$ & - & - & - & Drop object: left hand \\
\hline 9 & $44 / \mathrm{M}$ & $++/+++$ & $+/+++$ & +++ & ++ & C5-6 & Bil. forearm & $\begin{array}{l}\text { Finger jerks, sacral } \\
\text { sparing }\end{array}$ \\
\hline 10 & $76 / F$ & +++ & +++ & ++ & + & - & - & Gait disturbance \\
\hline 11 & $79 / \mathrm{F}$ & + & + & \pm & +++ & - & - & Marche., Warten. + \\
\hline 12 & $44 / \mathrm{M}$ & $+/-$ & $+/-$ & Inv./++ & ++ & - & $\begin{array}{l}\text { Right } \\
\text { U\&L/E }\end{array}$ & Inverted right triceps \\
\hline 13 & $50 / \mathrm{M}$ & +++ & +++ & +++ & ++ & - & - & $\begin{array}{l}\text { Finger jerks, ataxic } \\
\text { gait }\end{array}$ \\
\hline 14 & $47 / \mathrm{F}$ & ++ & ++ & ++ & - & - & Both hands & - \\
\hline 15 & $52 / \mathrm{M}$ & $+/ \pm$ & \pm & ++ & $\pm /++$ & - & Left hand & Finger jerks, Warten. + \\
\hline 16 & $63 / \mathrm{F}$ & - & - & ++ & +++ & - & - & Falling tendency \\
\hline 17 & $30 / \mathrm{F}$ & +++ & +++ & ++ & ++ & - & - & $\begin{array}{l}\text { Finger jerks, Warten. } \\
+, \text { W. }\end{array}$ \\
\hline 18 & $37 / \mathrm{M}$ & ++ & ++ & ++ & +++ & - & Left U\&L/E & Wartenberg -/+ \\
\hline 19 & $76 / \mathrm{M}$ & ++ & ++ & + & $++/+++$ & - & Feet & Vibration disturbance \\
\hline 20 & $33 / \mathrm{M}$ & +++ & +++ & +++ & +++ & - & All E. & - \\
\hline 21 & $73 / \mathrm{M}$ & $+++/+$ & ++ & Inv. & +++ & - & Both hands & $\begin{array}{l}\text { Inverted triceps } \\
\text { reflexes }\end{array}$ \\
\hline 22 & $40 / \mathrm{M}$ & +++ & +++ & ++ & +++ & - & Left hand & Finger jerks \\
\hline 23 & 39/M & \pm & \pm & +++ & +++ & C6 & $\begin{array}{l}\text { Right } \\
\text { U\&L/E }\end{array}$ & - \\
\hline 24 & $81 / \mathrm{M}$ & - & - & ++ & - & - & - & Neck \& shoulder pain \\
\hline
\end{tabular}

Brachi = brachioradialis; PTR = patellar tendon reflex; L/E = lower extremity; U/E = upper extremity; Inv. = inverted; Bil. = bilateral; Marche. $=$ Marche a petit pas; Warten. = Wartenberg; $\mathrm{E} .=$ extremities; $\mathrm{W}$. = weakness.

1 patient, C5-6 for 1 patient, C6 for 2 patients, and Th3 for 1 patient. In addition, 7 patients complained of tingling sensations, and for 3 of these patients, tingling sensations were localized to 1 hand. (Table 1, Fig. 1B, D).

\section{C5-6 intervertebral level myelopathy}

Twenty-four patients had C5-6 intervertebral level lesions (11 women and 13 men). Five patients $(20.8 \%)$ showed de- 


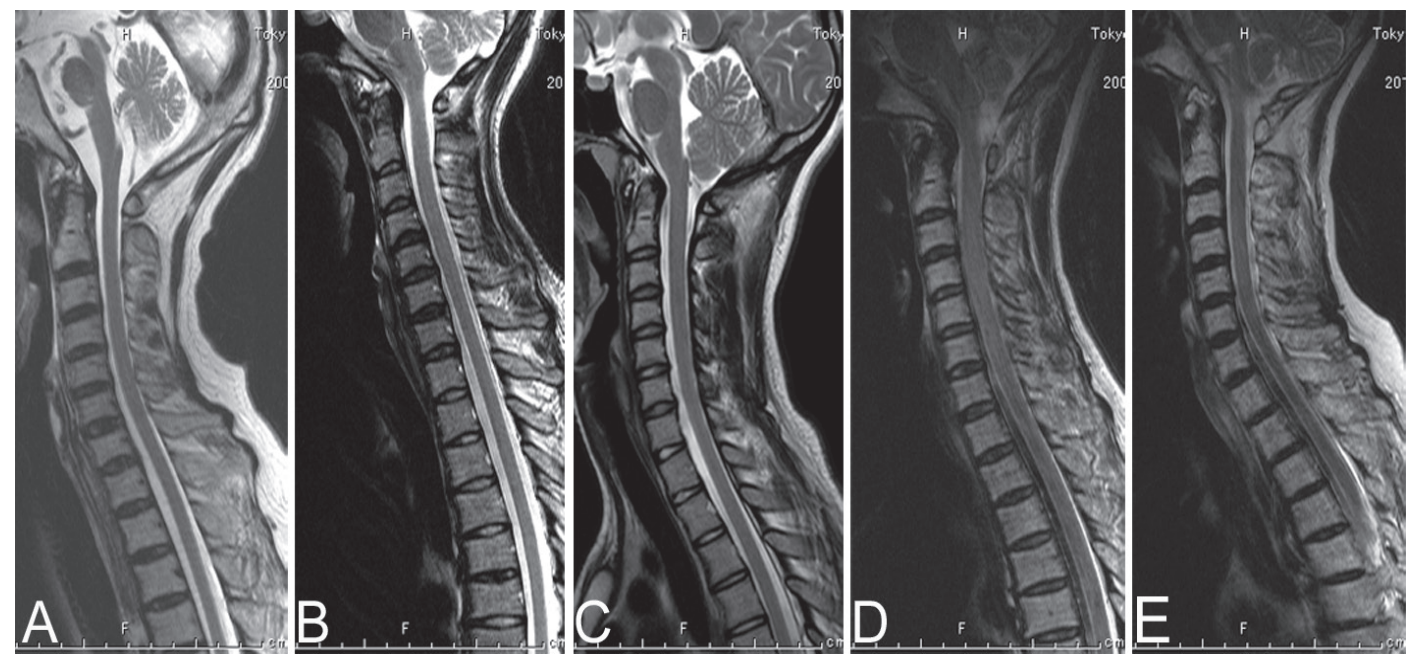

Figure 2. Sagittal T2-weighted magnetic resonance imaging of minimal change myelopathy patients (TR, 2400; TE, 120). (A) Patient 1: No apparent compression of the cervical spinal cord. (B) Patient 2: No apparent compression of the cervical spinal cord. (C) Patient 3: No apparent compression of the cervical spinal cord. (D) Patient 4: Narrow canal, but no apparent compression of the cervical spinal cord. (E) Patient 5: No apparent compression of the cervical spinal cord.

creased or absent triceps reflexes. Three patients $(12.5 \%)$ showed inverted triceps reflexes. Nine patients $(37.5 \%)$ showed decreased or absent biceps and brachioradialis reflexes. Six patients $(25 \%)$ showed bilateral finger jerks, which were elicited by the biceps and brachioradialis reflexes. Five of the 6 patients with finger jerks showed increased biceps and brachioradialis reflexes, while the remaining 1 patient showed decreased biceps and brachioradialis reflexes (inverted radial reflexes). Four patients (16.7\%) had sensory levels from C5-6 to C7: C5-6 for 1 patient, C6 for 1 patient, and $\mathrm{C} 7$ for 2 patients. Fourteen (58.3\%) patients complained of tingling sensations; of these, 4 had tingling sensations in the hands, with 2 patients having tingling sensations in both hands and 2 patients having tingling sensations in only 1 hand. (Table 2, Fig. 1A, C).

\section{C6-7 intervertebral level myelopathy}

Only 2 female patients had C6-7 intervertebral level lesions; of these, 1 patient had inverted triceps reflexes and the other patient complained of tingling sensations in all extremities.

\section{Minimal change myelopathy}

Five patients (4 women and 1 man) presented with neurological signs consistent with cervical compressive myelopathy but without any apparent lesions on the MRI (Fig. 2). One patient showed decreased biceps and brachioradialis reflexes, while 4 patients showed generalized hyperreflexia. Two patients showed finger jerks with increased biceps and brachioradialis reflexes. One patient showed Wartenberg reflexes. All patients had sensory levels from C5 to C7: C5 for
1 patient, C6 for 1 patient, C6-7 for 1 patient, and C7 for 2 patients. In addition, 1 patient showed bilateral dorsal interosseous muscle atrophy. (Table 3, Fig. 2).

\section{Discussion}

The following are the important findings of this study: 1) 4 of 12 patients (33.3\%) with C4-5 intervertebral level lesions showed generalized hyperreflexia; 2) 9 of 24 patients (37.5\%) with C5-6 intervertebral level lesions showed absent biceps and brachioradialis reflexes; 3) 6 of 24 patients (25\%) with C5-6 intervertebral level lesions showed bilateral finger jerks, with 5 patients showing increased and 1 patient showing decreased biceps and brachioradialis reflexes (inverted radial reflexes); and 4) 5 patients without any apparent MRI findings showed neurological signs consistent with cervical compressive myelopathy. The patellar reflexes were preserved or increased in most cases, except in those (usually elderly) patients who also had lumbar root lesions.

C3-4 intervertebral level myelopathy was only diagnosed in one patient. She showed generalized hyperreflexia, which was similar to that reported previous studies $[1,3,5]$. Because numb and clumsy hands due to $\mathrm{C} 4$ or $\mathrm{C} 5$ (C3-4 intervertebral level) lesions have been reported previously [9], objects dropping by the patient's left hand may be due to hand clumsiness and may represent a specific symptom of C3-4 intervertebral level cervical compressive myelopathy. The fasciculus cuneatus occupies most of the ventral aspect of the column adjacent to the commissure from the second to sixth cervical cord segment [9]. Clumsy hand due to C3-4 intervertebral level cervical compressive myelopathy may be 
Table 3. Clinical Characteristics of Minimal Change Myelopathy

\begin{tabular}{|c|c|c|c|c|c|c|c|c|}
\hline Patient & Age/Sex & Biceps & Brachi & Triceps & PTR & Level & Complaint & Others \\
\hline 1 & $75 / \mathrm{M}$ & $+++/++$ & $+++/++$ & ++ & - & $\mathrm{C} 7$ & Weakness L/E & $\begin{array}{l}\text { Bilateral DIO } \\
\text { atrophy }\end{array}$ \\
\hline 2 & $36 / F$ & $+++/++$ & +++ & +++ & +++ & C6 & Tingling Rt 2, $3 \mathrm{f}$. & $\begin{array}{l}\text { Finger jerks, } \\
\text { Warten. + }\end{array}$ \\
\hline 3 & $15 / \mathrm{F}$ & \pm & + & ++ & ++ & C6-7 & Pain of Rt U/E & - \\
\hline 4 & $57 / \mathrm{F}$ & +++ & +++ & +++ & +++ & $\mathrm{C} 7$ & $\begin{array}{l}\text { Tingling in both } \\
\mathrm{L} / \mathrm{E}\end{array}$ & $\begin{array}{l}\text { Finger jerks, } \\
\text { Vib. D. }\end{array}$ \\
\hline 5 & $68 / F$ & +++ & $+++/++$ & +++ & ++ & $\mathrm{C} 5$ & $\begin{array}{l}\text { Blindness of right } \\
\text { eye }\end{array}$ & - \\
\hline
\end{tabular}

Brachi $=$ brachioradialis; PTR = patellar tendon reflex; L/E = lower extremities; $\mathrm{DIO}=$ dorsal interosseus; Rt = right; $\mathrm{f} .=$ fingers; Warten. = Wartenberg; $\mathrm{U} / E=$ upper extremities; Vib. = vibration; $\mathrm{D} .=$ disturbance.

associated with this anatomical feature of fasciculus cuneatus.

The C4-5 intervertebral level corresponds to the C6 spinal cord segment, suggesting that C4-5 intervertebral level myelopathy must show C6 segmental signs. In this study, 5 patients $(41.7 \%)$ showed decreased biceps and brachioradialis reflexes corresponding to the $\mathrm{C} 6$ spinal cord segments. However, 4 of 12 patients (33.3\%) showed generalized hyperreflexia, and these findings correspond to the lesions above the C6 spinal cord segments. The sensory levels presented by $\mathrm{C} 4-5$ intervertebral level lesions in this study were C5, C5-6, C6, and Th3. These findings suggest that C4-5 intervertebral level lesions not only affect the C6 spinal cord segments but also affect the C5 spinal cord segments or another more rostral segment. Pre- and post-fixed brachial plexus anomalies have been described. The presence of intra- and extra-dural anastomoses anomalies might explain the variation in physical findings in patients with lesions at similar levels.

The C5-6 intervertebral level corresponds to the C7 spinal cord segment, suggesting that C5-6 intervertebral level myelopathy must show C7 segmental signs. In this study, 5 of 24 patients $(20.8 \%)$ showed decreased triceps reflexes, and 3 patients $(12.5 \%)$ showed inverted triceps reflexes. These findings correspond to the $\mathrm{C} 7$ spinal cord segments. However, 9 patients (37.5\%) showed decreased or absent biceps and brachioradialis reflexes, which correspond to the C6 spinal cord segments. The most probable explanation for this is C6-7 is the segment corresponding to the C5-6 intervertebral level. C5-6 intervertebral level lesions may cause both C7 myelopathies and C6 radiculopathies [4]. However, decreased or absent biceps and brachioradialis reflexes of present patients were usually bilateral. Present patients did not complain radicular pain. Therefore, explanation by complication of myelopathy and radiculopathy seems unlikely.

Six of the 24 C5-6 intervertebral level myelopathy patients $(25 \%)$ showed bilateral finger jerks, which were elicited by the biceps and brachioradialis reflexes; 5 of these 6 patients showed increased biceps and brachioradialis reflexes. The sensory levels were C5-6, C6, and C7. These findings also suggest that C5-6 intervertebral level lesions not only affect the $\mathrm{C} 7$ spinal cord segments but also affect the $\mathrm{C} 6$ spinal cord segments or may be caused by upward stimulation, namely, a caudal lesion that stimulates a more rostral segment.

One patient with a C6-7 intervertebral level lesion showed inverted triceps reflexes. Another patient complained of tingling sensations in all the extremities. These findings suggest that C6-7 intervertebral level lesions may affect the C7 spinal cord segments or another more rostral segment.

Five patients showed neurological signs consistent with cervical compressive myelopathy without any apparent lesions on the MRI. There may be such condition as congenitally based hyperreflexia, or this may be just a real deviation from what is considered normal. Some possible explanations for these findings may include psychosomatic disease, hysteria, or malingering. However, signs are the physical findings by clinicians, and there seem to be background changes corresponding to the signs. Although a usual MRI study may not be able to detect the lesions associated with these conditions, a dynamic MRI study with neck extension or flexion may reveal the lesions in these patients.

Previous studies have shown that the C4-5 intervertebral level corresponds to the C6 spinal cord segment, and 
the C5-6 intervertebral level corresponds to the C7 spinal cord segment $[1,3,4]$. However, another study has reported that C5-6 level lesions that manifested as dysesthesia of the thumb and index finger [5], with muscle stretch reflexes in the biceps and brachioradialis were diminished, whereas C6-7 level lesions that manifested dysesthesia of the middle fingers, with muscle stretch reflexes in the triceps were diminished [5]. Anatomically, C4-5 intervertebral level corresponds to the C5 segment, and C5-6 intervertebral level corresponds to C6-7 segment [13]. These anatomical findings match our results. Consequently, the C4-5 intervertebral level lesions not only affect the C6 spinal cord segments but also affect the $\mathrm{C} 5$ or another more rostral segment, and the C5-6 intervertebral level lesions not only affect the C7 spinal cord segment but also affect the $\mathrm{C} 6$ or another more rostral segment.

Although this study has provided valuable insight into the signs and symptoms important for level-specific diagnoses, it does have a few limitations. The number of patients with C3-4 and C6-7 intervertebral level lesions was small. The minimal change myelopathy patients did not undergo the dynamic MRI study. There was no age-matched control group. Generalized hyper- or hypo-reflexia may be not uncommon in normal subjects. Finger jerks may also be positive in normal subjects without other evidence of cervical myelopathy. Most of the patients only had mild or moderate myelopathy. However, because the number of patients studied here was considerable, we believe that the results of this study are reliable.

The C4-5 intervertebral level lesions can manifest as generalized hyperreflexia. The C4-5 intervertebral level lesions not only show $\mathrm{C} 6$ segment signs but also show $\mathrm{C} 5$ or other more rostral segment signs. Some patients with C5-6 intervertebral level lesions do not show any biceps and brachioradialis reflexes, and other patients can be characterized by finger jerks with increased biceps and brachioradialis reflexes. Thus, C5-6 intervertebral level lesions not only show C7 segment signs but also show C6 or other more rostral segment signs. Furthermore, some patients showed neurological signs consistent with cervical compressive myelopathy even though the MRI showed no apparent lesions.

\section{Acknowledgement}

We are grateful to nurses and co-medical staffs for helping our work.

\section{Conflict of Interests}

None.

\section{Grant Support}

None.

\section{References}

1. Seichi A, Takeshita K, Kawaguchi H, Matsudaira K, Higashikawa A, Ogata N, Nakamura K. Neurologic level diagnosis of cervical stenotic myelopathy. Spine (Phila Pa 1976). 2006;31(12):1338-1343.

2. Matsumoto $M$, Ishikawa $M$, Ishii $K$, Nishizawa $T$, Maruiwa H, Nakamura M, Chiba K, et al. Usefulness of neurological examination for diagnosis of the affected level in patients with cervical compressive myelopathy: prospective comparative study with radiological evaluation. J Neurosurg Spine. 2005;2(5):535-539.

3. Kokubun S. Neurological localization of the symptomatic level of lesion in cervical spondylotic myelopathy [in Japanese]. Rinsho Seikei Geka. 1984;19(4):417-424.

4. Ando T. Diagnosis and management of cervical spondylosis [in Japanese]. Rinsho Shinkeigaku. 2012;52(7):469479.

5. Yamazaki Y, Tachibana S, Yada K. Characteristic neurological signs in patients with cervical disc disease [in Japanese]. No Shinkei Geka. 1995;23(10):875-880.

6. Kasahata N. Bilateral finger jerks as a useful sign for diagnosis of cervical compressive myelopathy. J Neurol Res. 2011;1(1):22-29.

7. Adams KK, Jackson CE, Rauch RA, Hart SF, Kleinguenther RS, Barohn RJ. Cervical myelopathy with false localizing sensory levels. Arch Neurol. 1996;53(11):11551158 .

8. Simmons Z, Biller J, Beck DW, Keves W. Painless compressive cervical myelopathy with false localizing sensory findings. Spine (Phila Pa 1976). 1986;11(9):869-872.

9. Nakajima M, Hirayama K. Midcervical central cord syndrome: numb and clumsy hands due to midline cervical disc protrusion at the C3-4 intervertebral level. J Neurol Neurosurg Psychiatry. 1995;58(5):607-613.

10. Wells P. Cervical angina. Am Fam Physician. 1997;55(6):2262-2264.

11. Montgomery DM, Brower RS. Cervical spondylotic myelopathy. Clinical syndrome and natural history. Orthop Clin North Am. 1992;23(3):487-493.

12. Sung RD, Wang JC. Correlation between a positive Hoffmann's reflex and cervical pathology in asymptomatic individuals. Spine (Phila Pa 1976). 2001;26(1):6770.

13. Ropper AH, Samuels MA. Adams and Victor's Principles of Neurology, Ninth Edition. New York, McGrawHill. 2009;1186-1187. 